\title{
MPTC - A Minimum-Energy Path-Preserving Topology Control Algorithm for Wireless Sensor Networks*
}

\author{
Xian Zhou, Yun Li, Weiliang Zhao, Zhanjun Liu, and Qianbin Chen \\ Special Research Centre for Optical Internet \& Wireless Information Networks \\ Chongqing University of Posts \& Telecommunications \\ Chongqing, China, 400065 \\ zhouxian219@Gmail.com
}

\begin{abstract}
The topology control strategies of wireless sensor networks are very important to reduce the energy consumptions of sensor nodes and prolong the life-span of networks. In this paper, we put forward a minimum-energy pathpreserving topology control (MPTC) algorithm. MPTC not only resolves the problem of exceeding energy consumption because of the unclosed region in SMECN[2], but also preserves at least one minimum-energy path between every pair of nodes in a communication network. At last, we demonstrate the performance improvements of our algorithm through simulation.
\end{abstract}

Keywords: wireless sensor networks, topology control, minimum energy property, k-redundant edges.

\section{Introduction}

Wireless sensor network can be deployed in wide variety of civil and military applications. Because the power of sensor nodes are limited, so network protocols that minimize energy consumption is a major design goal for wireless sensor networks. As one of the key techniques, Topology control can design power-efficient algorithms that maintain network connectivity and optimize performance metrics such as network life and throughput.

At present, there are a number of documents carrying on the research on the topology control [3],[4],[5],[6],[7],[8]. The work most closely related to ours is that of $\mathrm{Li}$ (Erran) Li et al. [2], they propose an effective topology control algorithm, which is called a small minimum-energy communication network (SMECN). But SMECN has a flaw about the unclosed region which could result in exceeding energy consumption. We put forward a new topology control algorithm- Minimum-energy Path-preserving Topology Control (MPTC) which not only resolves the problem, but also ensures the connectivity of network which has minimum-energy path property.

Supported by the Research Grants by the Science \& Tech. Commission of Chongqing (CST2006BB2370), the Science and Technology Research Project of Chongqing Municipal Education Commission of China (KJ070521), and Ph.D Grand of CQUPT (A2007-07). 


\section{Model}

We assume that all nodes in the wireless sensor network are deployed in twodimensional area, where no two nodes are in the same physical location. Each node knows its own location, and has a unique identification code. A transmission between node $u$ and $v$ takes power $p(u, v)=t d(u, v)^{n}$ for some appropriate constant $t$, where $n \geq 2$ is the path-loss exponent of outdoor radio propagation models [1], and $d(u, v)$ is the distance between $u$ and $v$. In this paper, we designate $n$ equals 4 .

When each node of the network uses its biggest power in working, this topology is called the biggest power topology $\left(G_{\max }\right)$. A $\operatorname{PATH}(u, v)=\left(u=u_{0}, \cdots u_{n}=v\right)$ in $G$, the consumed energy when messages delivered through this path is $C(\operatorname{PATH}(u, v))=n c+\sum_{i=0}^{n-1} P\left(u_{i}, u_{i+1}\right), c$ is the consumed energy when a node receives messages.

\section{The MPTC Algorithm}

According to the SMECN[2], the direct-transmission regions(DTR) of the nodes can be divided two different types: closed region and unclosed region. If $u$ can find its transmitted power $p_{u}\left(p_{\max }>p_{u}>0\right)$ that satisfies $F\left(u, p_{u}\right) \supseteq \eta$, u's DTR is a closed region. (Fig. 1(a) shows closed region). Otherwise, $F\left(u, p_{u}\right) \nsupseteq \eta$ when $p_{u}\left(p_{\max }>p_{u}>0\right)$, u's DTR is a unclosed region. (Fig .1(b) shows unclosed region.). The DTR of the nodes at the verge of the wireless network are almost unclosed region. SMECN will set $p_{\max }$ as the node's transmitted power when the node's DTR is an unclosed region, which makes these nodes consume more energy.

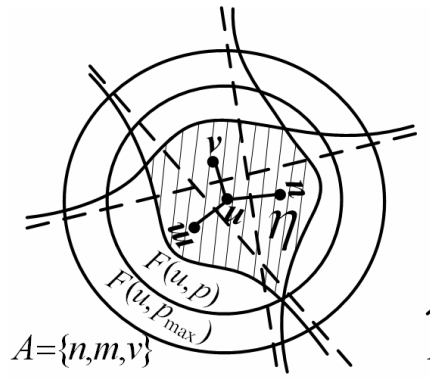

(a) Closed region

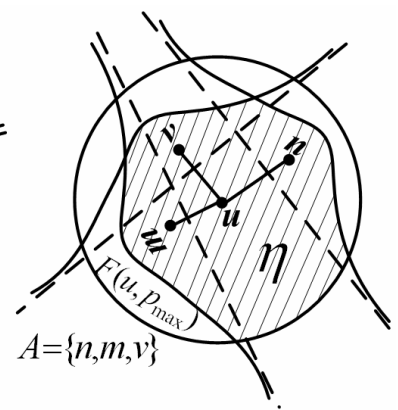

(b) Unclosed region

Fig. 1. Closed region and unclosed region

Different from SMECN[2], MPCT sets the transmitted power of nodes without judging whether the nodes' transmission region covers its direct- transmission region. 
The detail of MPTC algorithm is shown in Fig.2, which consists of the following main steps.

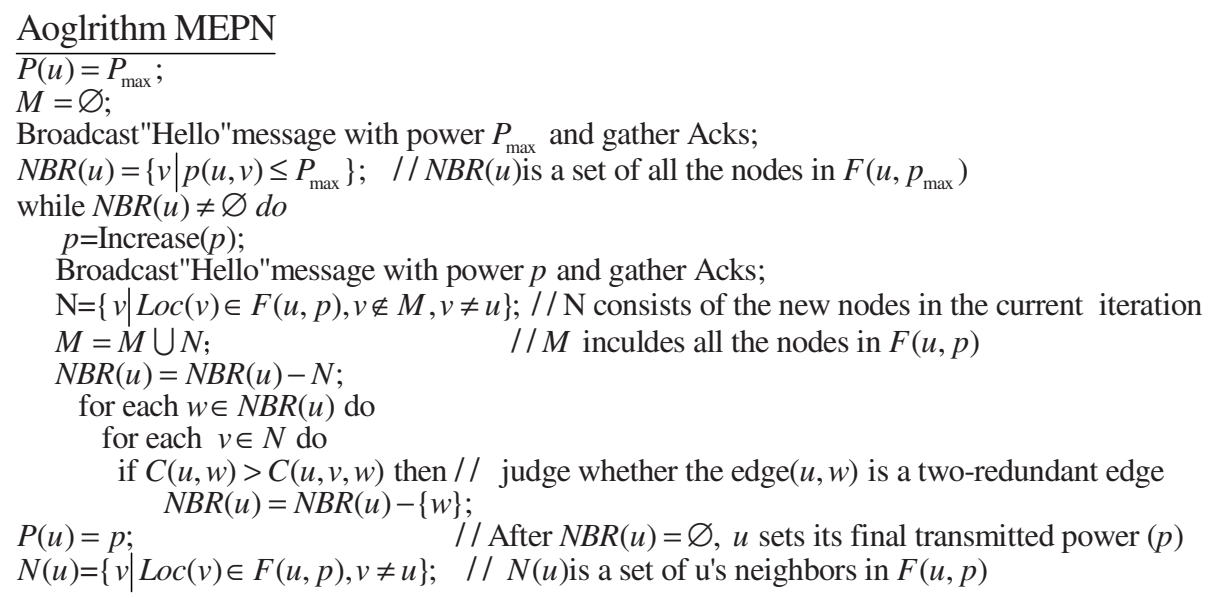

Fig. 2. Algorithm MPTC running at node $u$

Define the graph $G=(V, E)$ by taking $(u, v) \in E$ iff $v \in \operatorname{Nbr}(u)$, as constructed by the algorithm in Fig.2. Thus, the following theorem holds.

Theorem 1. If the $G_{\max }$ can ensure the connectivity of the wireless network, then the subgraph $G=(V, E)$ can also ensure the connectivity of network and has the minimum-energy property [2].

Proof- Connectivity: We assume that there is a $\operatorname{PATH}(u, v)=\left(u=u_{0}, \ldots, u_{n}=v\right)$ in $G_{\max }$. According to MPTC algorithm, if $\operatorname{edge}\left(u_{i}, u_{i+1}\right)$ is not a two-redundant edge[2], we reserve the this edge in $G$. Otherwise, if $\operatorname{edge}\left(u_{i}, u_{i+1}\right)$ is a tworedundant edge, there are $\operatorname{PATH}^{*}\left(u_{i}, u_{i+1}\right)=\left(u_{i}, w_{i}^{1}, \cdots, w_{i}^{m}, u_{i+1}\right)$ in $G$, and $\mathrm{C}\left(\operatorname{PATH}^{*}\left(u_{i}, u_{i+1}\right)\right)<\mathrm{C}\left(\operatorname{edge}\left(u_{i}, u_{i+1}\right)\right)$. So, if $u_{i}$ and $u_{i+1}$ are connected in $G_{\max }$, they are also connected in $G$. We can find a path like $\operatorname{PATH}^{*}(u, v)=\left(u=u_{0}, w_{0}^{1}, \cdots, w_{0}^{m_{0}}, u_{1}, w_{1}^{1}, \cdots, w_{1}^{m_{1}}, \cdots, u_{n-1}, w_{n-1}^{2}, \cdots, w_{n-1}^{m_{n-1}}, u_{n}=v\right) \mathrm{i}$ $\mathrm{n} G$. The above process prove that for a given pair of nodes $u$ and $v$, if there is a path between them in $G_{\max }$, there is also a path between $u$ and $v$ in $G$. Therefore, if $G_{\max }$ is connected, so is $G$.

Proof- The minimum-energy property: We assume there is a minimum-energy path between $u$ and $v$ in $G_{\max }, \operatorname{PATH}(u, v)=\left(u=u_{0}, \ldots, u_{n}=v\right)$, which is not in $G$. There 
is at least an edge $\left(u_{i}, u_{i+1}\right)(i=(0, \cdots, n-1))$ in $\operatorname{PATH}(u, v)$ which is not included in $G$. Due to the connectivity of $G$, there is another $\operatorname{PATH}^{*}\left(u_{i}, u_{i+1}\right)=\left(u_{i}, w_{i}^{1}, \cdots, w_{i}^{m}, u_{i+1}\right)$ in $G$ to connect $u_{i}$ and $u_{i+1}$. According to MPTC algorithm, it is not hard to show $C\left(\operatorname{PATH}^{*}\left(u_{i}, u_{i+1}\right)\right)<C\left(\operatorname{edge}\left(u_{i}, u_{i+1}\right)\right)$, so there is another $\operatorname{PATH}^{*}(u, v)=\left(u=u_{0}, \cdots, u_{i}, w_{i}^{1}, \cdots, w_{i}^{m}, u_{i+1}, \cdots, u_{n}=v\right)$ in $G_{\text {max }}$ and $C(\operatorname{PATH}(u, v))>C\left(\operatorname{PATH}^{*}(u, v)\right)$, which means that $\operatorname{PATH}(u, v)$ is not a minimum-energy path between $u$ and $v$ in $G_{\max }$. It is contrary to the foregoing assumption. Hence we can conclude $G$ has the minimum-energy path property.

\section{Evaluations}

\section{A. Simulation Environment}

The simulation is conducted on simulator NS-2[9]. We generated 10 random network with 100 nodes. The nodes are randomly placed in a rectangular region of $1000 \mathrm{~m} \times 1000 \mathrm{~m}$. Each node has a maximum transmission range of $200 \mathrm{~m}$ and is equipped with 20J of energy at beginning of the simulation. We randomly set $4 \mathrm{CBR}$ flows at sending rate of 5 packets/sec, each packet size is 512 bytes. We use AODV[10] as routing protocol.

\section{B. Simulation Results}

We firstly concentrate our research on topology structure. Fig. 3 shows the networks' topology structure controlled by MaxPower, SMECN and MPTC respectively. Compared with the subnetworks controlled by SMECN and MPTC, the MPTC's subnetwork is smaller than that of SMECN, which can decrease the collision and save energy of nodes.

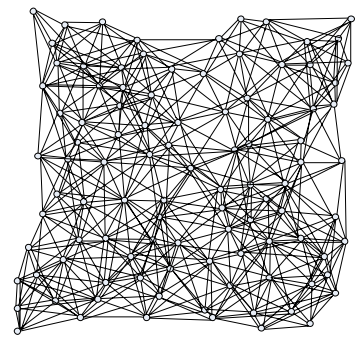

(a) MaxPower

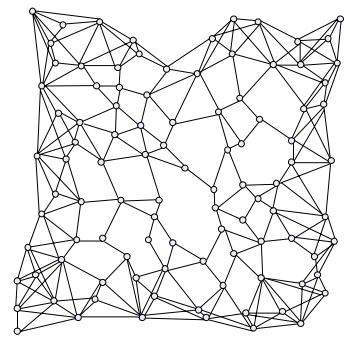

(b) SMECN

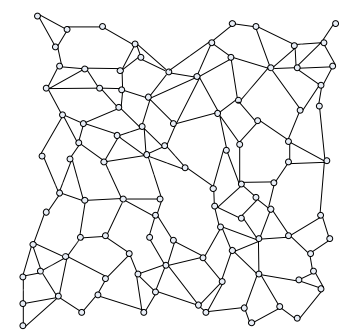

(c) MPTC

Fig. 3. The topology structure controlled by MaxPower, SMECN and MPTC

We simulate the average remaining energy of nodes in the path of flows after 300s simulation time. Fig.4(a) shows the simulation results for MPTC, SMECN and 
MaxPower, respectively. We can see that the average energy decrease rate of MPTC is significantly lower that that of SMECN and MaxPower Fig.4(b) shows the average degree of nodes for MPTC, SMECN and MaxPower, respectively. From Fig.4(b), we can conclude the average degree of MPTC is evidently lower than that of SMECN. The average degree of MPTC is only 70\% 75\% of SMECN. After about 270s, the average degree of MaxPower is decrease to 0 , which means the most nodes in the network have no neighbor because a lot of nodes have been dead.

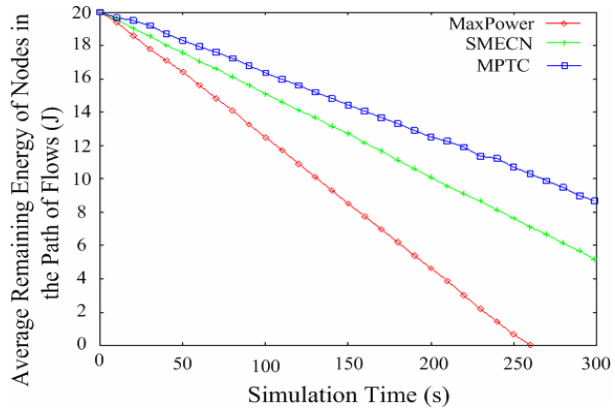

(a)

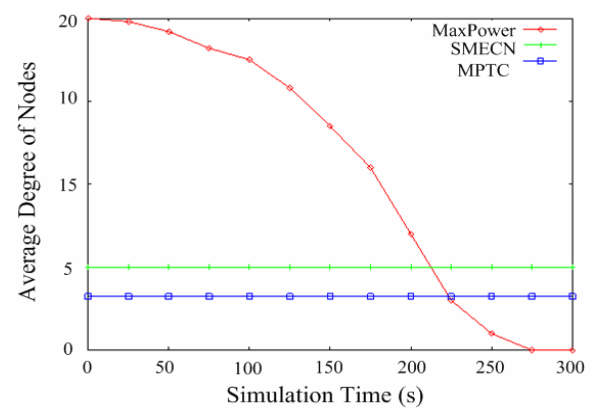

(b)

Fig. 4. (a) Average remaining energy of nodes (b) Average degree of nodes

\section{Conclusion}

Topology control can reduce the consumptions of sensor nodes, optimize the performance of networks and prolong the life span of the networks through adjusting the transmission power of sensor nodes. Our paper puts forward a new topology control algorithm of wireless sensor networks-MPTC. It not only avoids the exceeding energy consumption which results from the unclosed region in SMECN, but also ensures the connectivity of network which has minimum-energy path property. Finally, we have shown the performance improvements of MPTC over SMECN and MaxPower through simulation.

\section{References}

1. Rappaport, T.S.: Wireless Communications: Principles and Practice. Prentice-Hall, Englewood Cliffs[M], NJ (1996)

2. Erran, L., Halpern, J.Y.: A Minimum-Energy Preserving Topology-Control Algorithm[J]. IEEE Transaction on Wireless Communications 3(3), 910-921 (2004)

3. Erran, L., Halpern, J.Y., Bahl, P.: A Cone-Based Distributed Topology-Control Algorithm for Wireless Multi-Hop Networks[J]. IEEE/ACM Transactions on Networking 13(1), 147 159 (2005)

4. Yin, B., Shi, H., Shang, Y.: A Two-Level Strategy for Topology Control in Wireless Sensor Networks[A]. In: ICPADS 2005. Proceedings of 11th International Conference on Parallel and Distributed Systems, pp. 358-362 (2005) 
5. Chen, B., Jamieson, K., Balakrishnan, H., Morris, R.: Span: An energy-efficient coordination algorithm for topology maintenance in ad hoc wireless networks[J]. Mobile Computing and Networking 8(5), 85-96 (2002)

6. Liu, J., Li, B.: Distributed topology control in wireless sensor networks with asymmetric links[J]. IEEE GLOBECOM 2003 3(8), 1257-1262 (2003)

7. Busse, M., Haenselmann, T., Effelsberg, W.: A Topology and Energy Control Algorithm for Wireless Sensor Networks [A]. In: MSWiM 2006, pp. 317-321 (2006)

8. Rodoplu, V., Meng, T.H.: Minimum energy mobile wireless networks. IEEE J. Select. Areas Commun. 17, 1333-1344 (1999)

9. VINT Project. The UCB/LBNL/VINT Network Simulator-ns (Version2). [Online], http://www.isi.edu/nsnam/ns

10. Perkins, C.E., Royer, E.M.: Ad-hoc on-demand distance vector routing. In: 2nd IEEE Workshop Mobile Computing Systems Applicat, pp. 90-100 (1999) 